\title{
Introduction to the thematic issue on Affect Aware Ubiquitious Computing
}

\author{
Faiyaz Doctor ${ }^{\mathrm{a}, *}$, Rahat Iqbal ${ }^{\mathrm{a}}$ and Victor Zamudio ${ }^{\mathrm{b}}$ \\ ${ }^{a}$ Department of Computing, Faculty of Engineering and Computing, Coventry University, United Kingdom \\ ${ }^{\mathrm{b}}$ Division of Graduate Studies and Research, Instituto Tecnológico de León, México
}

\section{Introduction}

The growth of embedded and networked enabled physical devices collectively termed as the 'Internet of Things' (IoT) has become an enabler for facilitating richer context awareness, personalisation through integration of intelligence, into everyday consumer devices. The global IoT market is expected to hit $\$ 7.1$ trillion by 2020 (IDC) and is projected to drive the circulation and use of some 50 billion connected devices. This influx of personal, mobile and wearable devices will open up many new research opportunities focusing on human centered technology with a particular focus on extending the contextual sensing and smart processing capabilities of ubiquitous systems and smart environments. Consequentially there will be need for researchers and industry to develop more unobtrusive and natural communication between computing artefacts and users to make pervasive systems more aware and reactive to their feelings, moods and desires.

Affective computing (AC) is concerned with emotional interactions performed with and through computers. It is defined as "computing that relates to, arises from, or deliberately influences emotions" (R. Picard, MIT Media Lab). Practical applications of Affective Computing (AC) based systems seek to achieve a positive impact on our everyday lives by monitoring, recognising and acting on our emotions, physiological signals, speech, facial expressions and gestures. Integrating these sensing modalities into intelligent pervasive computing systems will reveal a far richer picture of how our fleeting emotional responses, changing moods, feelings and sensations, such as pain, touch,

*Corresponding author. Email: faiyaz.doctor@coventry.ac.uk. flavours and smells, are a reaction to or influence how we implicitly or explicitly interact with the environment and computing artefacts within.

This thematic issue on affect aware ubiquitous computing aims to showcase recent research within the Ambient Intelligence (AmI) and pervasive computing community that focuses on how $\mathrm{AC}$ can be used in novel systems for eliciting, and interpreting different affective information modalities in context of user behaviours and interaction in smart environments. This has resulted in developing new forms of affective interactions, mediation and transference between humans and the digital environment where devices recognise and respond to the emotional state of user to create interesting affective systems that enhance the user's experiences and enable the environment to interact and connect with the user in novel and interesting ways. Research in the area has needed to address challenges in multimodal sensor fusion for affect elicitation; approaches for modelling and recognition of physiological inputs, user behaviour, cognation and the development of novel affect responsive interfaces and control. In parallel this has also necessitated the development of pervasive and mobile devices, networking and infrastructural frameworks (e.g., cellular, ad hoc, hybrid) for managing and integrating affective information in smart environments.

The Guest editors of this JAISE thematic issue, Faiyaz Doctor, Rahat Iqbal and Victor Zamudio have endeavoured to make this a relevant, timely and interesting collection of articles for the ambient intelligence and smart environments community. The thematic issue received a total of 16 submissions from which 6 high quality papers were selected based on double blind review process. This is a rapidly growing area of research and the issue only shows a selected 
spectrum of the work being done in this area. We look forward to hearing from readers about other areas and problems that need to be addressed as we develop better ways of using affective data pervasively and in context of different, diverse data sources to create highly complex, information rich scenarios.

\section{In this thematic issue}

In the paper 'An Extensible Platform for Seamless Integration and Management of Applications for Emotion Sensing and Interpretation' the authors introduce an extensible software platform based on a middleware architecture that provides developers with uniform interfaces and services enabling affect aware applications to access the results from existing (or newly implemented) tools for emotion sensing and interpretation. The system called Vikara provides components that make it possible for platform managers to visually monitor affective states as they are sensed by the various available tools.

The paper titled 'An Affective Ephemeral Social Network for Vehicular Scenarios' presents the idea of an affective system in which a network architecture is proposed for individual affective profiling, social matching and social group management in the context of heavy traffic environments. The network aims to create a space for communication between vehicles to elicit user identities, personalities and preferences and enabling users to convey affective cues.

The cognitive load placed on users by the proactive and spontaneous provisioning of service functionality as a response to their interactions in ambient intelligent environments can be mentally burdening. The paper titled 'Cognitive Resource-Aware Unobtrusive Service Provisioning in Ambient Intelligence Environments' examines this issue though the development of cognitive-resource-aware activity and service description models derived from cognitive psychology. The authors propose a dynamic service binding and scheduling mechanism based on different types of interferences and their mental resource demand requirements.
The paper titled 'Unobtrusive Emotion Sensing and Interpretation in Smart Environment' presents a smart environment of web services that has been developed to integrate and manage different new and existing emotion sensing applications, which working together to provide tracking and recognition of human affective states in real time. Emotion interpreters based on recognition of facial features have been developed where fuzzy systems are used for quantitative measurement and recognition of intensity levels for basic and non-prototypical facial expressions.

Due to an aging population there is an increasing number of elderly people living independently which can result in social isolation. The problem of loneliness in older adults is addressed in the paper titled 'Inferring Loneliness Levels in Older Adults from Smartphones'. Here the authors present predictive models to determine the level of loneliness related to different health and social aspects which are focused around user behaviours and activities that can be monitored using a Smartphone.

Finally the paper titled 'Mediating Individual Affective Experience through the Emotional Photo Frame' proposes an emotional photo frame system that dynamically controls digital photograph setting based on a user's affective states and their specified emotion reaction rules. The system is designed to help users recognize and convey emotional experiences with other users through the recognition and transformation of physiological responses into personalized visual and aural elements on the photograph. The research also suggests how emotions can be mediated through artifacts and how emotional experiences can be shared between users.

\section{Acknowledgments}

The guest editors wish to thank all the reviewers for their valuable contributions to this thematic issue. In addition, we gratefully acknowledge the support and guidance of the editorial staff of JAISE. Finally, we would like to express our huge appreciation to all the authors for their high-quality submissions that made this thematic issue possible. 